\title{
Correction to: The Strategic Biomarker Roadmap for the validation of Alzheimer's diagnostic biomarkers: methodological update
}

\author{
Marina Boccardi ${ }^{1,2}$. Alessandra Dodich ${ }^{3,4}$ - Emiliano Albanese ${ }^{5}$. Angèle Gayet-Ageron ${ }^{6} \cdot$ Cristina Festari $^{7}$. \\ Nicholas J. Ashton ${ }^{8,9,10,11}$. Gérard N. Bischof ${ }^{12} \cdot$ Konstantinos Chiotis $^{13,14}$. Antoine Leuzy ${ }^{13}$. Emma E. Wolters ${ }^{15}$. \\ Martin Walter ${ }^{16}$. Gil D. Rabinovici ${ }^{17}$ - Maria Carrillo ${ }^{18}$. Alexander Drzezga ${ }^{19,20,21}$. Oskar Hansson ${ }^{22,23}$. \\ Agneta Nordberg ${ }^{13,24} \cdot$ Rik Ossenkoppele $^{25,26} \cdot$ Victor L. Villemagne $^{27,28} \cdot$ Bengt Winblad $^{24,29} \cdot$ Giovanni Frisoni $^{2,30}$. \\ Valentina Garibotto ${ }^{4,16}$
}

Published online: 21 September 2021

(c) The Author(s) 2021

\section{Correction to: Eur J Nucl Med Mol Imaging https://doi.org/10.1007/s00259-020-05120-2}

The authors regret Table 2 needs to be rearranged so that it will be easier to be understood.

This article is part of the Topical Collection on Erratum

The original article can be found online at https://doi.org/10.1007/ s00259-020-05120-2.

Marina Boccardi

marina.boccardi@dzne.de

1 German Center for Neurodegenerative Diseases (DZNE), Rostock, Germany

2 LANVIE, Laboratory of Neuroimaging of Aging, University of Geneva, Geneva, Switzerland

3 Center for Neurocognitive Rehabilitation (CeRiN), CIMeC, University of Trento, Trento, Italy

4 NIMTlab, Neuroimaging and Innovative Molecular Tracers Laboratory, University of Geneva, Geneva, Switzerland

5 USI, Università della Svizzera Italiana, Lugano, Switzerland

6 Clinical Epidemiology Department, University of Geneva, Geneva, Switzerland

7 LANE, Laboratory of Alzheimer's Neuroimaging and Epidemiology, IRCCS Istituto Centro San Giovanni di Dio Fatebenefratelli, Brescia, Italy

8 Wallenberg Centre for Molecular and Translational Medicine, University of Gothenburg, Gothenburg, Sweden
The corrected Table appears below.

9 Department of Psychiatry and Neurochemistry, Institute of Neuroscience \& Physiology, the Sahlgrenska Academy at the University of Gothenburg, Mölndal, Sweden

10 NIHR Biomedical Research Centre for Mental Health and Biomedical Research Unit for Dementia at South London and Maudsley NHS Foundation, London, United Kingdom

11 Institute of Psychiatry, Psychology \& Neuroscience, King's College London, London, United Kingdom

12 Department of Nuclear Medicine, University Hospital Cologne, Cologne, Germany

13 Division of Clinical Geriatrics, Center for Alzheimer Research, Department of Neurobiology, Care Sciences and Society, Karolinska Institutet, Stockholm, Sweden

14 Theme Neurology, Karolinska University Hospital, Stockholm, Sweden

15 Alzheimer Center Amsterdam, Department of Neurology, Amsterdam Neuroscience, Vrije Universiteit Amsterdam, Amsterdam UMC, Amsterdam, Netherlands

16 Nuclear Medicine and Molecular Division, Geneva Medical Hospital, Geneva, Switzerland

17 Departments of Neurology, Radiology \& Biomedical Imaging, University of California, San Francisco, USA

18 Alzheimer's Association, Chicago, IL, USA 
19 University of Cologne, Faculty of Medicine, Cologne, Germany

20 German Center for Neurodegenerative Diseases (DZNE), Bonn/Cologne, Germany

21 Institute of Neuroscience and Medicine (INM-2), Molecular Organization of the Brain, Research Center Jülich, Jülich, Germany

22 Clinical Memory Rresearch Unit, Department of Clinical Sciences Malmö, Lund University, Lund, Sweden

23 Memory Clinic, Skåne University Hospital, Malmö, Sweden

24 Karolinska University Hospital, Theme Aging, Geriatric Clinic, Huddinge, Sweden

25 Alzheimer Center Amsterdam, Department of Neurology, Amsterdam Neuroscience, Vrije Universiteit Amsterdam, Amsterdam UMC, Amsterdam, Netherlands
26 Department of Clinical Memory Research, Lund University, Lund, Sweden

27 Department of Molecular Imaging \& Therapy, Austin Health, Melbourne, VIC, Australia

28 Department of Medicine, Austin Health, The University of Melbourne, Melbourne, VIC, Australia

29 Department of Neurobiology, Care Sciences and Society, Division of Neurogeriatrics, Karolinska Institutet, Stockholm, Sweden

30 Memory Clinic, University Hospital, Geneva, Switzerland 


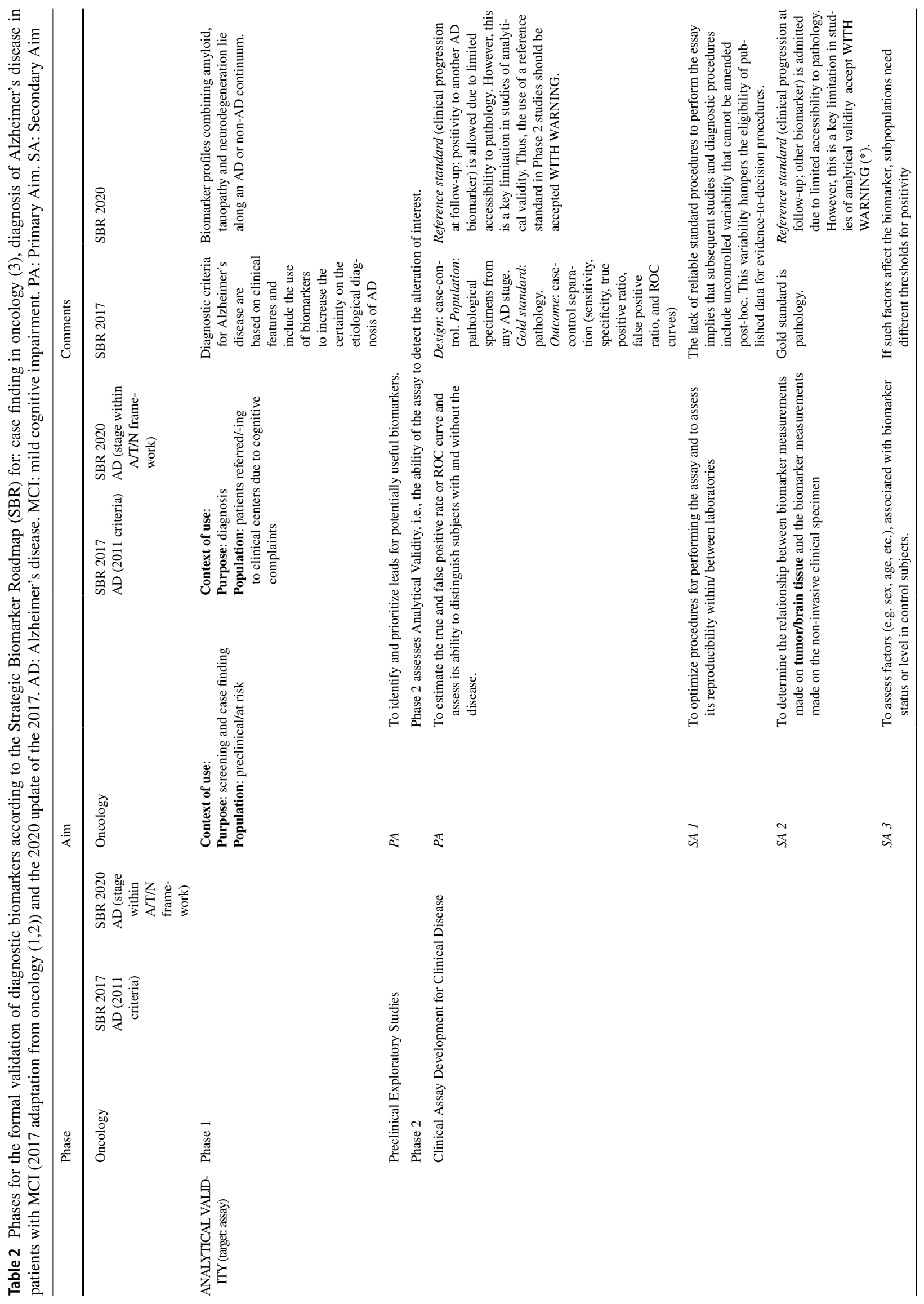




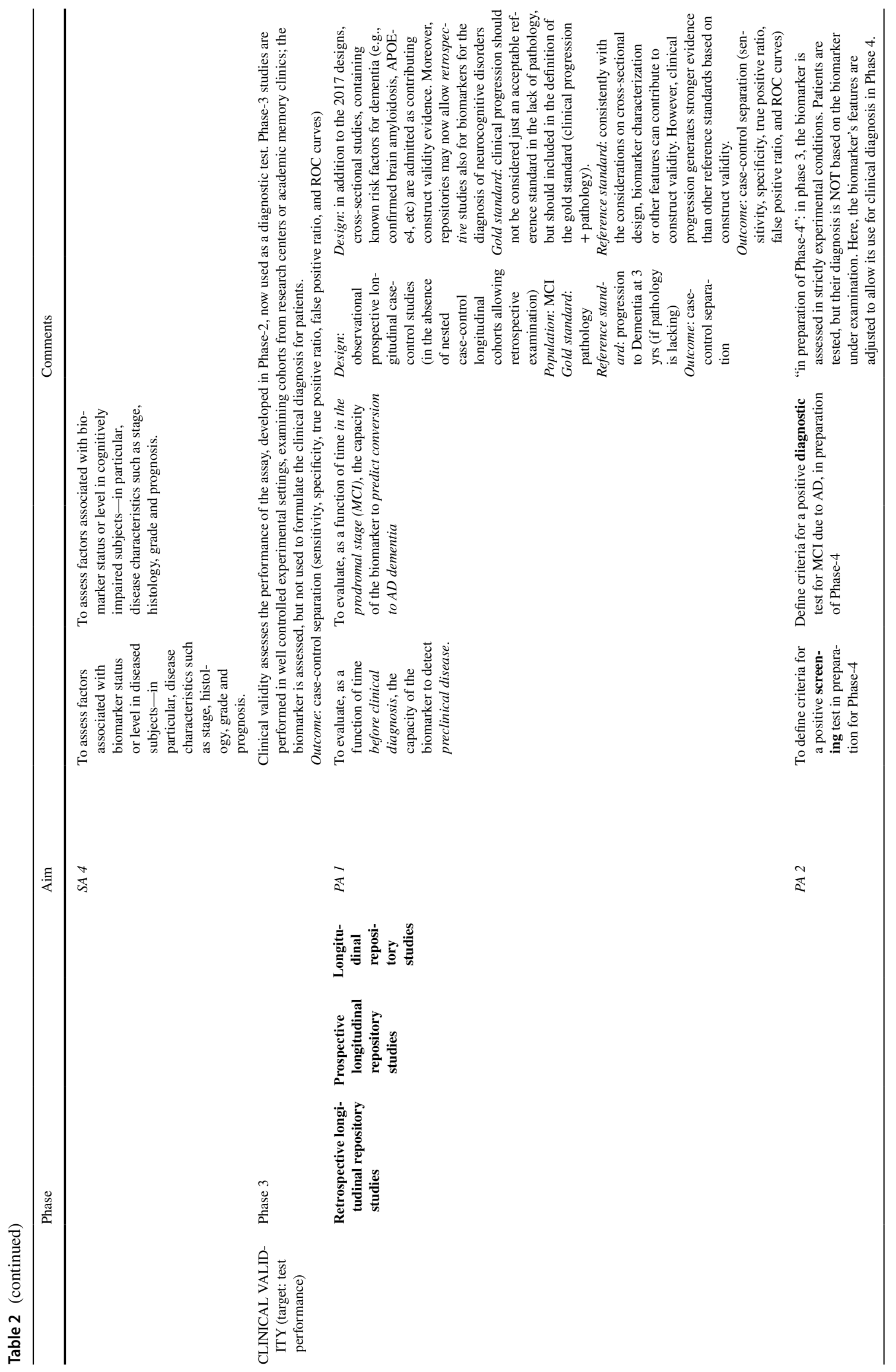




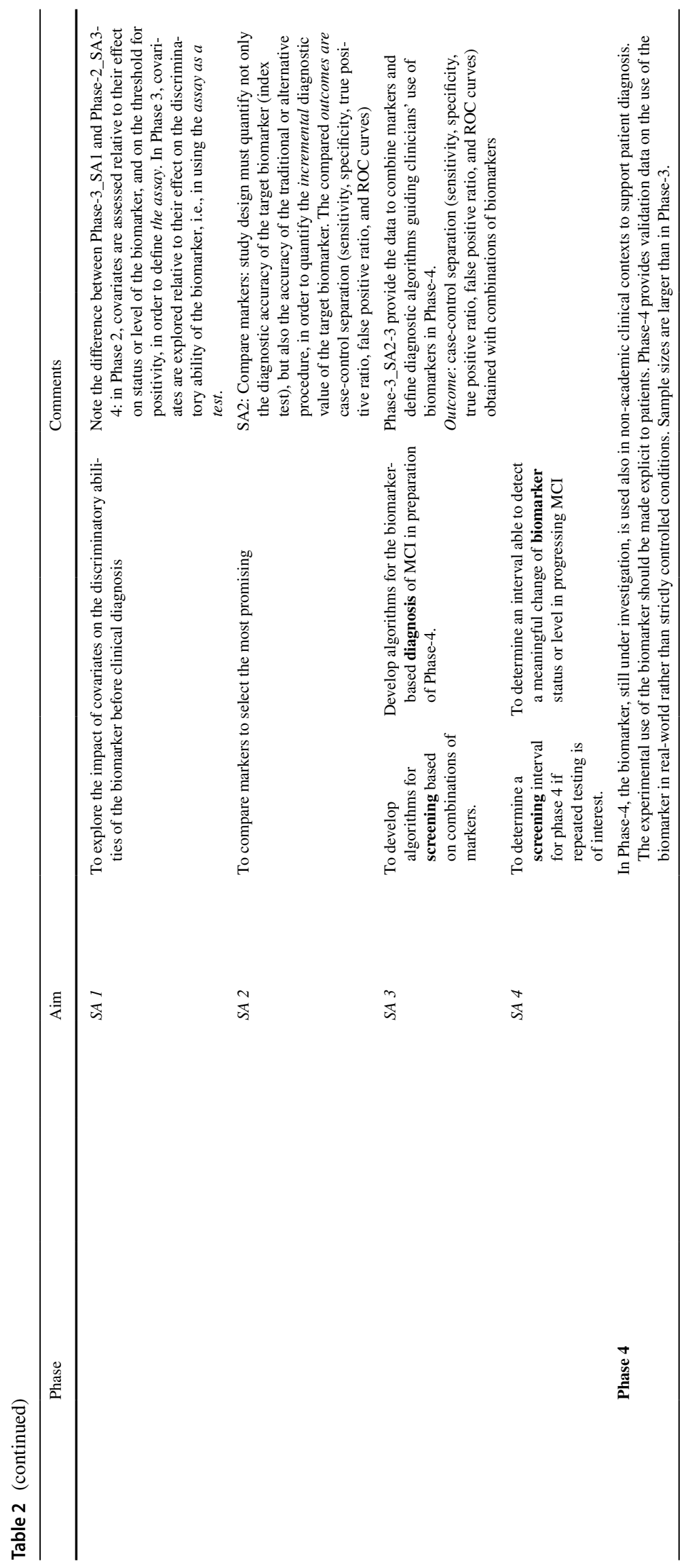




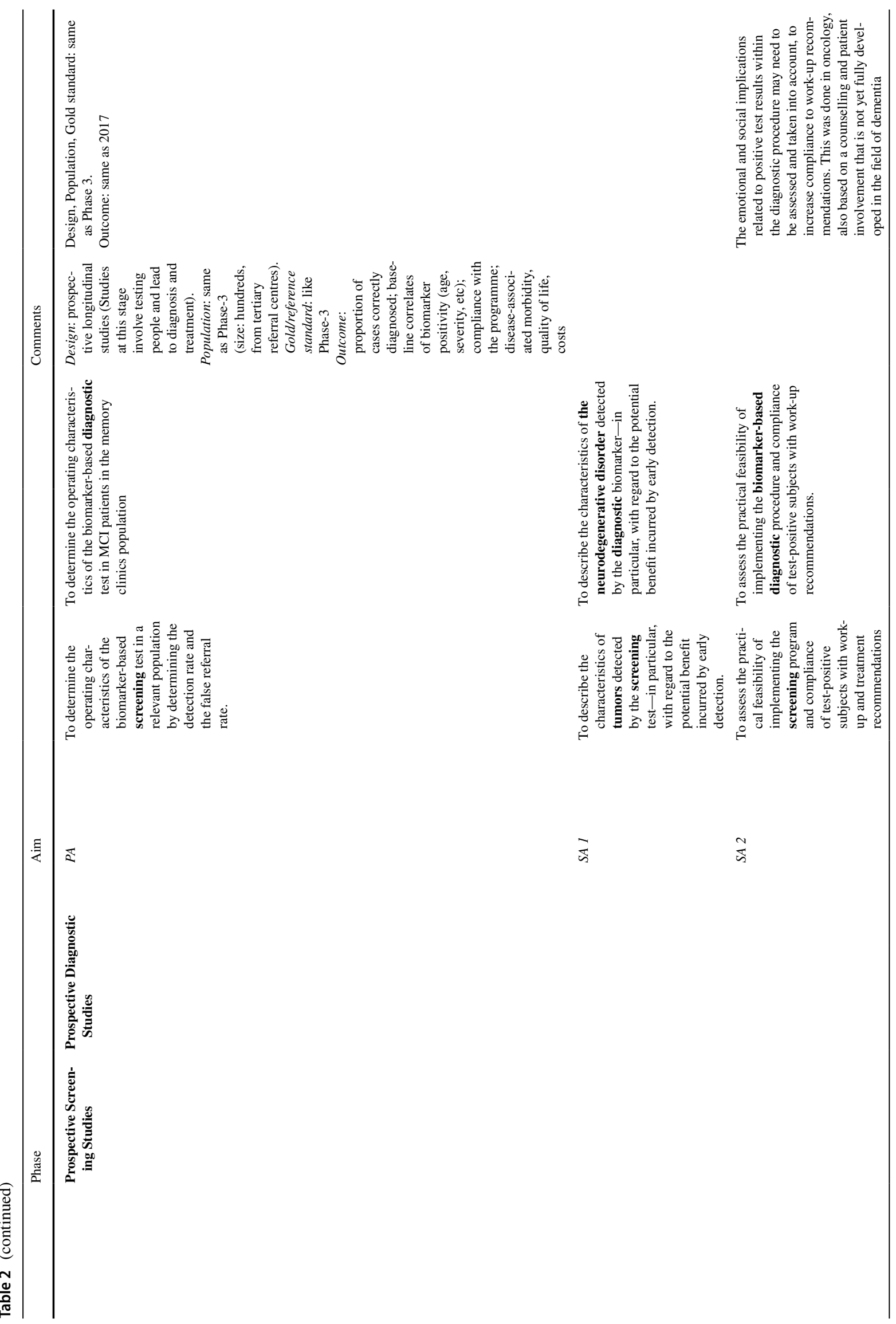




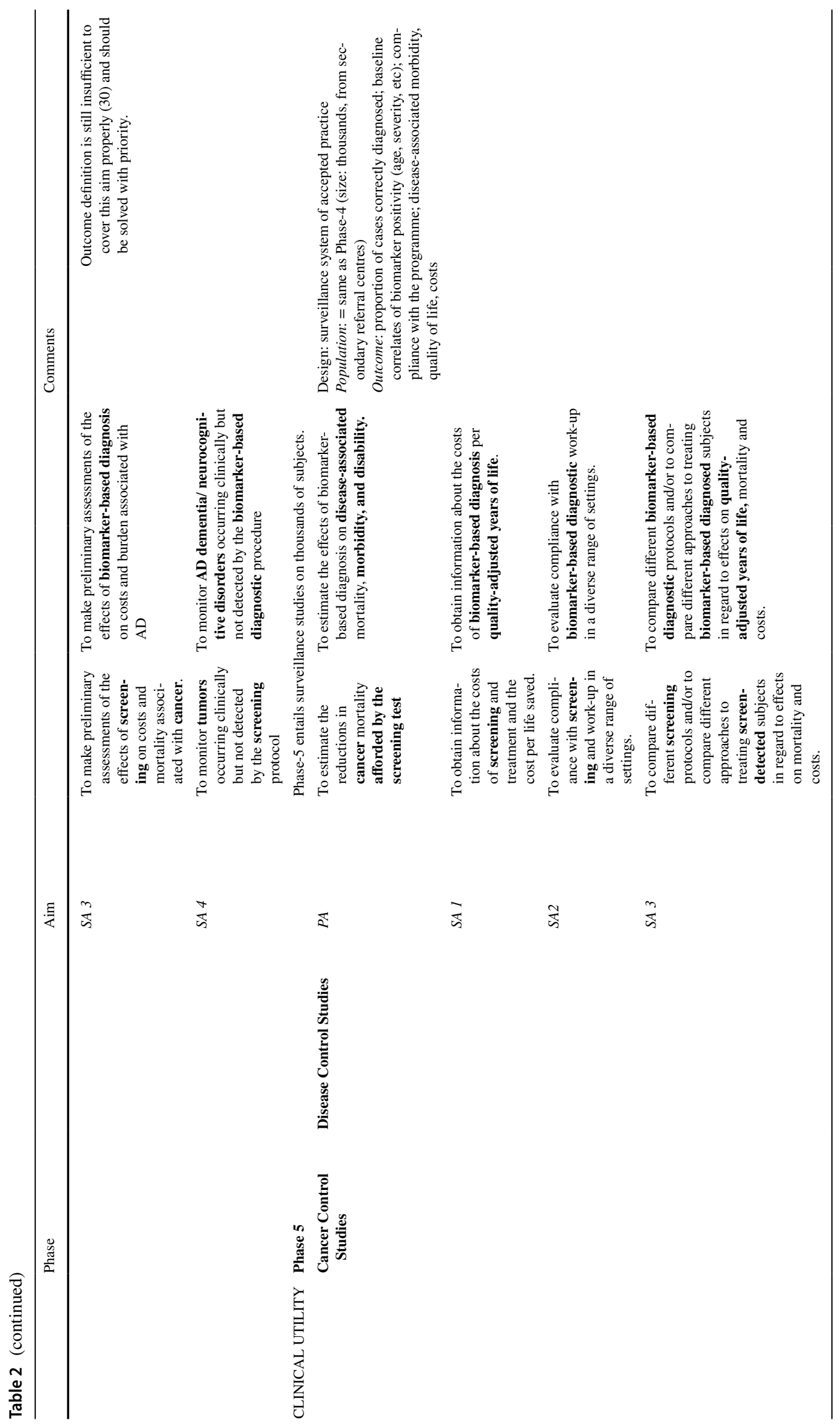

